\title{
Research on Complexity Evaluation of Complex Products and Systems Based on Fuzzy Comprehensive Evaluation Method
}

\author{
Wang Xiuhong ${ }^{1, a}$, Suo Jing ${ }^{2, b}$ \\ 1,2 School of Management Engineering, Zhengzhou University of Aeronautics, China \\ a wangzzia@163.com; 22248562793@qq.com
}

\begin{abstract}
Keywords: Complex Products and Systems; Fuzzy Comprehensive Evaluation Method; Complexity evaluation
\end{abstract}

\begin{abstract}
High complexity is the typical characteristic of Complex products and systems (CoPS). Complexity increases risk of CoPS research, and complexity is the key factor which results in failure of CoPS research. Evaluation to CoPS complexity makes researchers find the complex grades of all parts so that researchers take more time focus on the key parts. On the basis of internal structure and external characteristic of CoPS research, from the perspective of system the article sets evaluation index system of CoPS complexity which include external environment complexity, technology intensive, and organization complexity. Fuzzy comprehensive evaluation method is used to evaluate its complexity. According to the evaluation results, enterprise can reduce the management risk as much as possible. We hope to provide beneficial help for the CoPS development practice.
\end{abstract}

\section{Introduction}

Complex products and systems (CoPS) usually refers to some higher development costs, technology intensive, customers have specific requirements of small batch or single product and service, etc. Such as intelligent transportation systems[1]. The research and development ability of CoPS is a main sign to measure a nation's overall innovation ability even international competitiveness [2]. The success of CoPS development can lead to the embedded technology to be applied in other industries, and even lead to the upgrading of the industrial chain.

Complexity is the typical characteristics of CoPS, the more complex of the product means that stronger of product performance, harder to control the subsystem and an increasing number of form factors. CoPS is often to satisfy personalized customer demand, and always accompanies a high investment risk. It usually needs to sign a contract with customers, and according to the order's requirement to produce. Different CoPS project generally do not have similarity, so it is inappropriate to have mass production, but always take a single production mode. In the process of design and manufacture, the active communication among users, equipment suppliers and other partners is needed to reduce development risk and cost [3]. Because of the CoPS complexity and technology intensive, there are some unpredictable events often occurring in the process of design and manufacture. Therefore, carrying on the corresponding management by means of evaluating its complexity to determine the level of complexity, which is a key of reducing risk.

\section{Complexities and the Complexity of CoPS}

Complexity usually refers to the length of the computer language when describing a certain thing. It is generally acknowledged that describing a thing own the longer length of the computer 
language, indicating the thing is more complicated[4]. At present, some scholars have studied evaluating complexity problem of CoPS, and they research from different angle to study the CoPS complexity evaluation problem. Browning and Eppinger in the paper of Modeling impacts of process architecture on cost and schedule risk in product development mentioned that companies successfully manage complexity, meaning they will enjoy a competitive advantage in the market[5]. The defense industry relies heavily on systems engineering (SE) processes to help manage complexity and risk. In this research they present novel methods for improving complexity and risk management that are consistent with current systems engineering practices[6] . Gokpinar in the article of The Impact of Misalignment of Organizational Structure and Product Architecture on Quality in Complex Product Development considered that the complexity assessment also facilitates an analysis of the misalignment that may exist between the organization and product structures[7]. Du Shengpin has studied on the complexity of rapid transit system and pointed out that the greater the complexity value of the driveway and mixed area, the higher complexity it would be [8]. .Liu wei brings in the research of measuring complexity when studying the model of complex information system, which is used to set up the complexity of the data model, and by citing two practical enterprise for its validation[9].

But we think the CoPS innovation is one system engineering, and is usually made up of many highly specialized, closely linked and different components in the field of technology, and its performance is highly dependent on its components and structure. Therefore, it is very appropriate to evaluate the complexity of CoPS innovation from the perspective of system.

\section{CoPS innovation system structure}

In order to reduce the production risk as much as possible in the process of management, then it must explore the enterprise's complex products and systems in-depth research. CoPS innovation system structure diagram is shown in Fig 1.

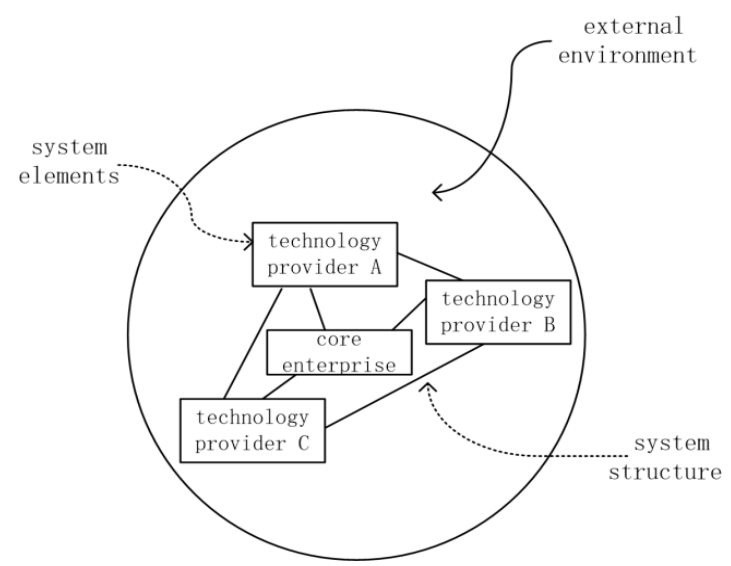

Fig.1. CoPS innovation system structure

Compared with the ordinary mass products, CoPS innovation system summarizes the characteristics of complex products and systems in CoPS external environment complexity, technology intensive and organization complexity. Because of product own customization demand, so the technical requirement is very strict, and the complexity of the technology determines whether the function of the product can meet customer's requirements. However, there is some restriction and influence in technology between each other as well. The technical department or the manufacturers need to cooperate with each other. For the organization complexity, including the top leaders of enterprises, employees, customers, suppliers, etc. whom should be considered in production design when they participate in this various organizations. It also requires organizations to coordinate with each other, and constantly improves the structure of the system. Of course, in 
CoPS innovation system, when the external environment condition changed, then the system will change too. Such as, the funding quantity increase or decrease, and it will have influence on the performance of the product or technology, so it is very important for the influence of external factors.

Therefore, this article mainly focuses on that three aspects of innovation system, and take it as a base point to discuss about building the CoPS complexity index, and then hoping it can avoid the risk in the process, and enables enterprise could survive in a more favorable condition.

\section{Building the CoPS complexity evaluation index system}

The establishment of evaluation index system is the key to whether the evaluation work could go smoothly, and whether the index system is reasonable will directly relate to the scientific of the evaluation model and veracity of the evaluation results.

Based on System structure shown in Fig 1, which contains the CoPS three first-level indicators that are the CoPS external environment complexity, technology intensive and organization complexity, and based on the analysis for CoPS innovation system structure, according to the characteristics of the first-level index, and then further divide into 14 secondary indicators to study the complexity in detail, the detail is shown in Fig 2.

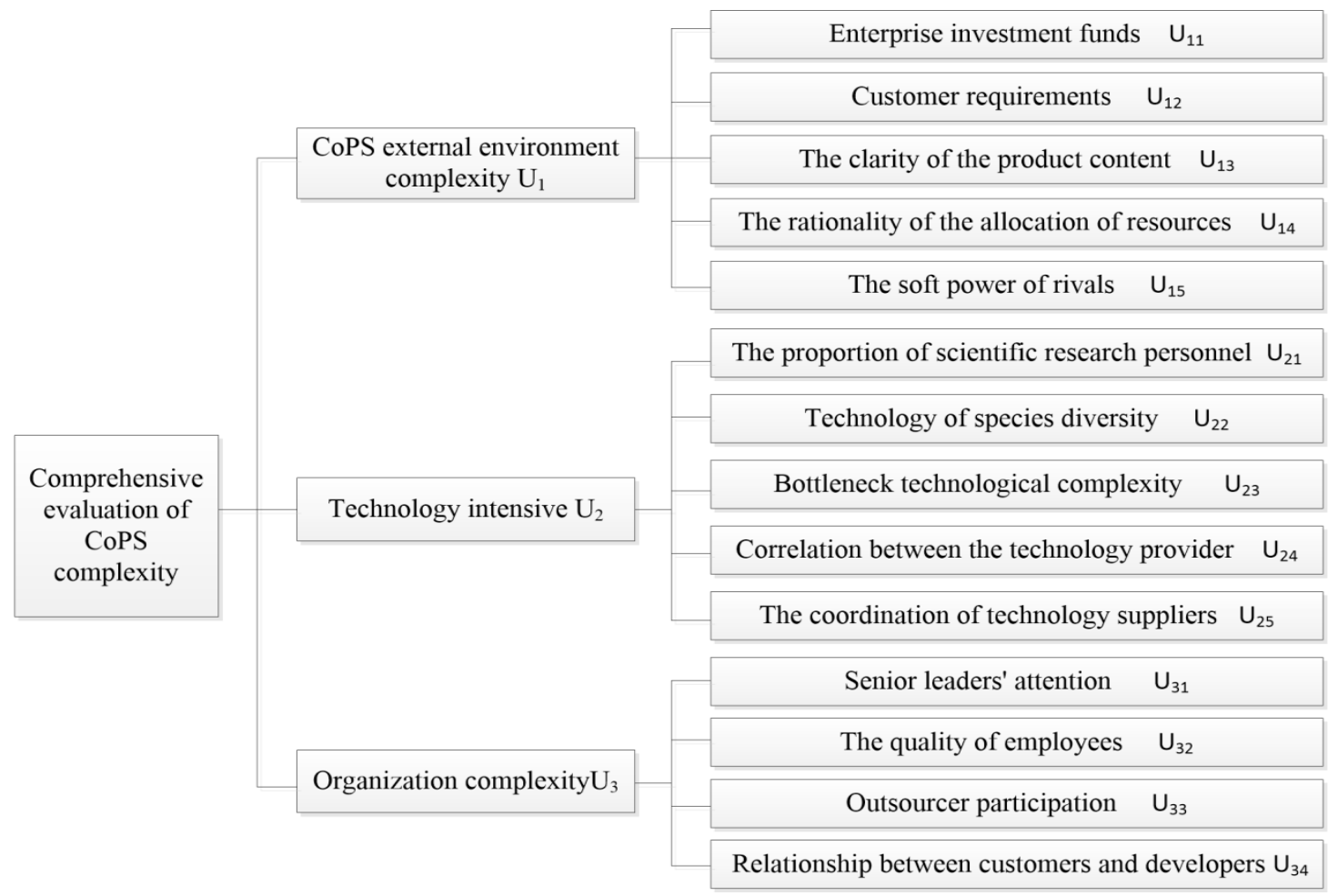

Fig 2. Evaluation index system of CoPS complexity

\section{Case analyses}

According to the complex products and systems of evaluation index shown in Fig 1, this paper adopts the secondary fuzzy comprehensive evaluation method to evaluate, steps are as follows: 
$U=\left(\begin{array}{lll}U_{1} & U_{2} & U_{3}\end{array}\right)$

Set the first-level indicators:

$U_{1}=\left(\begin{array}{lllll}U_{11} & U_{12} & U_{13} & U_{14} & U_{15}\end{array}\right)$

$U_{2}=\left(\begin{array}{llllll}U_{21} & U_{22} & U_{23} & U_{24} & U_{25}\end{array}\right)$

$U_{3}=\left(\begin{array}{llll}U_{31} & U_{32} & U_{33} & U_{34}\end{array}\right)$

as the second-level indicators. Evaluation sets:

$$
\begin{aligned}
& V=\left(\begin{array}{llll}
V_{1} & V_{2} & V_{3} & V_{4}
\end{array}\right) \\
& =\left(\begin{array}{llll}
\text { high } & \text { higher } & \text { medium } & \text { low }
\end{array}\right)
\end{aligned}
$$

Determine the membership degree and weight Fuzzy relationship matrix $\mathrm{R}$ (membership degree matrix), and the weight are the important influence factors. Setting weights and membership degree must be fair and objective, which mainly depend on the influence of factors in the enterprise and expert's subjective understanding for indicators [10].

Use the fuzzy comprehensive evaluation method to solve problem A company in Zhengzhou as an example to study the CoPS complexity, and the expert evaluation method is adopted in this paper, through expert consultation to build the judgment matrix, after the consistency check, and then get the index weight. There are 10 experts grade to this several indicators, and for each indicator statistic can get the membership degree, if there are four experts comment for high, the high membership degree is 0.4 , and so on. So the CoPS complexity evaluation and membership degree is shown in Tab.1.

From the table 1, the first layer:

$$
a_{0}=\left(\begin{array}{lll}
U_{1} & U_{2} & U_{3}
\end{array}\right)=\left(\begin{array}{lll}
0.25 & 0.4 & 0.35
\end{array}\right)
$$

In the same way, the second layer:

$$
\begin{aligned}
& a_{1}=\left(\begin{array}{lllll}
0.3 & 0.25 & 0.15 & 0.1 & 0.2
\end{array}\right) \\
& a_{2}=\left(\begin{array}{lllll}
0.2 & 0.25 & 0.1 & 0.3 & 0.15
\end{array}\right) \\
& a_{3}=\left(\begin{array}{llll}
0.35 & 0.2 & 0.3 & 0.15
\end{array}\right)
\end{aligned}
$$




$$
\begin{aligned}
& b_{1}=a_{1} r_{1} \\
& =\left(\begin{array}{lllll}
0.3 & 0.25 & 0.15 & 0.1 & 0.2
\end{array}\right)\left(\begin{array}{cccc}
0.4 & 0.4 & 0.2 & 0 \\
0.3 & 0.4 & 0.2 & 0.1 \\
0.1 & 0.3 & 0.4 & 0.2 \\
0.2 & 0.5 & 0.3 & 0 \\
0.2 & 0.3 & 0.4 & 0.1
\end{array}\right) \\
& =\left(\begin{array}{llll}
0.3 & 0.3 & 0.2 & 0.15
\end{array}\right)
\end{aligned}
$$$$
b_{2}=a_{2} r_{2}=\left(\begin{array}{llll}
0.2 & 0.3 & 0.25 & 0.2
\end{array}\right)
$$$$
b_{3}=a_{3} r_{3}=\left(\begin{array}{llll}
0.2 & 0.3 & 0.35 & 0.2
\end{array}\right)
$$

Get the standardized data:

$$
\begin{aligned}
& b_{1}=\left(\begin{array}{llll}
0.316 & 0.316 & 0.210 & 0.158
\end{array}\right) \\
& b_{2}=\left(\begin{array}{llll}
0.210 & 0.316 & 0.264 & 0.210
\end{array}\right) \\
& b_{3}=\left(\begin{array}{llll}
0.190 & 0.286 & 0.334 & 0.190
\end{array}\right)
\end{aligned}
$$

\begin{tabular}{|c|c|c|c|c|c|c|c|}
\hline \multicolumn{8}{|c|}{ CoPS of this enterprise complexity evaluation and membership degree } \\
\hline Target layer & The first layer & The second layer & weight & high & higher & medium & low \\
\hline \multirow{14}{*}{$\begin{array}{l}\text { CoPS of this } \\
\text { enterprise } \\
\text { complexity } \\
\text { evaluation } \\
\text { system }\end{array}$} & \multirow{5}{*}{$\begin{array}{c}\text { CoPS external } \\
\text { environment } \\
\text { complexity } \\
0.25\end{array}$} & Enterprise investment funds & 0.3 & 0.4 & 0.4 & 0.2 & 0 \\
\hline & & Customer requirements & 0.25 & 0.3 & 0.4 & 0.2 & 0.1 \\
\hline & & $\begin{array}{l}\text { The clarity of the product } \\
\text { content }\end{array}$ & 0.15 & 0.1 & 0.3 & 0.4 & 0.2 \\
\hline & & $\begin{array}{l}\text { The rationality of the } \\
\text { allocation of resources }\end{array}$ & 0.1 & 0.2 & 0.5 & 0.3 & 0 \\
\hline & & The soft power of rivals & 0.2 & 0.2 & 0.3 & 0.4 & 0.1 \\
\hline & \multirow{5}{*}{$\begin{array}{c}\text { Technology } \\
\text { intensive } 0.4\end{array}$} & $\begin{array}{l}\text { The proportion of scientific } \\
\text { research personnel }\end{array}$ & 0.2 & 0.2 & 0.5 & 0.1 & 0.2 \\
\hline & & $\begin{array}{c}\text { Technology of species } \\
\text { diversity }\end{array}$ & 0.25 & 0.1 & 0.4 & 0.4 & 0.1 \\
\hline & & $\begin{array}{l}\text { Bottleneck technological } \\
\text { complexity }\end{array}$ & 0.1 & 0.3 & 0.4 & 0.3 & 0 \\
\hline & & $\begin{array}{l}\text { Correlation between the } \\
\text { technology provider }\end{array}$ & 0.3 & 0.2 & 0.5 & 0.1 & 0.2 \\
\hline & & $\begin{array}{l}\text { The coordination of } \\
\text { technology suppliers }\end{array}$ & 0.15 & 0.1 & 0.3 & 0.5 & 0.1 \\
\hline & \multirow{4}{*}{$\begin{array}{c}\text { Organization } \\
\text { complexity } \\
0.35\end{array}$} & Senior leaders' attention & 0.35 & 0.1 & 0.3 & 0.4 & 0.2 \\
\hline & & The quality of employees & 0.2 & 0.3 & 0.5 & 0.2 & 0 \\
\hline & & Outsourcer participation & 0.3 & 0.2 & 0.3 & 0.3 & 0.2 \\
\hline & & $\begin{array}{c}\text { Relationship between } \\
\text { customers and developers }\end{array}$ & 0.15 & 0.2 & 0.4 & 0.3 & 0.1 \\
\hline
\end{tabular}

Tab 1. CoPS complexity evaluation and membership degree 


$$
\begin{aligned}
& r_{0}=\left(\begin{array}{lll}
b_{1} & b_{2} & b_{3}
\end{array}\right)^{T} \\
& =\left(\begin{array}{llll}
0.316 & 0.316 & 0.210 & 0.158 \\
0.210 & 0.316 & 0.264 & 0.210 \\
0.190 & 0.286 & 0.334 & 0.190
\end{array}\right) \\
& b_{0}=a_{0} r_{0} \\
& =\left(\begin{array}{lll}
0.3 & 0.4 & 0.3
\end{array}\right)\left(\begin{array}{lllll}
0.316 & 0.316 & 0.210 & 0.158 \\
0.210 & 0.316 & 0.264 & 0.210 \\
0.190 & 0.286 & 0.334 & 0.190
\end{array}\right) \\
& =\left(\begin{array}{llll}
0.25 & 0.316 & 0.334 & 0.210
\end{array}\right)
\end{aligned}
$$

Based on the principle of maximum membership degree, 0.334 is the maximum value, which is corresponding to the $\mathrm{V}$ set in medium level. So it is concluded that the enterprise of the complexity of the CoPS is medium.

\section{Conclusion}

This fuzzy comprehensive evaluation model take the expert group to grade to overcome the single factor scores, too subjective or speculation factors influence on the evaluation results, which to a large extent solve the problem of the CoPS in complexity research. Therefore, the secondary fuzzy comprehensive evaluation method provides an effective quantitative analysis model for the complexity of the CoPS research.

In this paper, based on the research of complex products and systems, by using fuzzy comprehensive evaluation method to establish the perfect evaluation system and evaluate enterprise risk investment, so the top decision makers would have a good grasp of enterprise management, and reduce the risk of the enterprise investment. For the scholars to study the complex product system complexity on the theory and method choice has played a certain significant function.

\section{Acknowledgment}

Thanks for the fund provided Humanities and social science project of Henan Provincial Department of Education (Grant No.2015-GH-039)and Key scientific research projects of Henan high school (Grant No.16A630063)

\section{References}

[1] Hansen K L, Rush H. Hotspots in complex product systems Emerging issues in innovation management

[J]. Technovation, 1998, 18(8/9):555-561.

[2] Zou Shuliang,Chen Jiahua,Liu Bing.A Study on Virtual Enterprise Model Faced to The Complex Product Systems:with Example from NPP Item[J]. Journal of Nanhua University(Social Science Edition),2005,01:20-25.

[3] Lu Zihua,Song Jianqiu,Zhang Meng.Supplier Selection and Evaluation Index System Based on Borda-Choice Method[J] Science and Technology Management Research,2015,07:192-197.

[4] Du Juan.Biosignal Variability Analysis and Its Application in The Evolution of Hypertension[D]. Zhe Jiang University,2006.

[5] Browning, T.R. and S.D. Eppinger. 2002. Modeling impacts of process architecture on cost and schedule risk in product development. Engineering Management, IEEE Transactions on, 49(4): 428-442.

[6] Group, I.S.H.W. 2010. Systems Engineering Handbook. January 2010 Vol. v. 32. San Diego: International Counsel on Systems Engineering. 374.

[7] Gokpinar,B.,W.J. Hopp, and S.M.R. Iravani. 2010. The Impact of Misalignment of Organizational Structure and Product Architecture on Quality in Complex Product Development. Management Science, 
56(3): 468-484.

[8] Du Shengpin, Ma Yongfeng. Quantitative Analysis on Complexity of Weaving Segment with Conflicts [J]. Journal of Transportation Systems Engineering and Information Technology, 2014, 01:53-58.

[9] Liu Wei, Ge Shilun, Wang Nianxin, Yin Jun.Measuring. The Complexity of Information System Based on The Date Complexity[J]. Systems Engineering-Theory\&Practice,2013,12:3198-3208.

[10] Zhao Chunchang, Tao Li,Pan Rui.Application of Fuzzy Synthetic Evaluation on Polity Decision[J]. Journal of Shenyang Arch.and Giv.Eng.Univ. (Natural Science), 2001,04:318-320. 